OPEN ACCESS

Edited by:

David E. MacHugh,

University College Dublin, Ireland

Reviewed by:

Shahin Eghbalsaied,

Islamic Azad University, Isfahan, Iran

Wenguang Liu,

South China Sea Institute of

Oceanology (CAS), China

*Correspondence:

Shiduo Sun

SSdsm@Tom.com

Specialty section:

This article was submitted to

Livestock Genomics,

a section of the journal

Frontiers in Genetics

Received: 17 June 2019 Accepted: 23 September 2019

Published: 15 November 2019

Citation:

Xu G, Zhang H, LiX, Hu J, Yang $G$ and Sun $S$ (2019) Genome-

Wide Differential Expression

Profiling of Ovarian circRNAs Associated With Litter Size in Pigs.

Front. Genet. 10:1010.

doi: 10.3389/fgene.2019.01010

\section{Genome-Wide Differential Expression Profiling of Ovarian circRNAs Associated With Litter Size in Pigs}

\author{
Gaoxiao Xu ${ }^{1,2}$, Huifang Zhang ${ }^{1}$, Xiao $\mathrm{Li}^{1}$, Jianhong Hu ${ }^{1}$, Gongshe Yang ${ }^{1}$ and Shiduo Sun ${ }^{1 *}$ \\ ${ }^{1}$ Key Laboratory of Animal Genetics, Breeding and Reproduction of Shaanxi Province, College of Animal Sciences and \\ Technologies, Northwest A\&F University, Yangling, China, 2 Teaching and Research Section of Biotechnology, Nanning \\ University, Nanning, China
}

Circular RNAs (circRNAs) have been emerging as an important regulator in mammalian reproduction via acting as miRNA sponges. However, the circRNAs in porcine ovaries related with litter size remains largely unknown. In this study, porcine ovaries with smaller or larger litter size (LLS) were subjected to high-throughput RNA sequencing. In total, 38,722 circRNAs were identified, of which 1,291 circRNAs were commonly expressed in all samples. There were 56 circRNAs significantly down-regulated and 54 circRNAs up-regulated in LLS pig ( $\mid \log 2$ (fold change) $\mid>1$, FDR < 0.05). Bioinformatics predicted that most of circRNAs harbored miRNA binding sites, and the expression patterns of circRNAs and their putative binding miRNAs were validated by $\mathrm{qPCR}$. Moreover, the expression of circ-TCP11/miR-183 was significantly reversely correlated and their direct interaction was confirmed by dual-luciferase assay. Our study indicates that circRNAs may play potential effects on modulating porcine litter size.

Keywords: circRNA, ovary, litter size, pig, miRNA

\section{INTRODUCTION}

Increasing litter size has been a global goal for pig breeders and producers, and larger litter size plus shorter farrowing intervals are desperately expected to expand piglets per sow per year, which is the predominant force to boost economic success of sow husbandry (Zak et al., 2017; Kemp et al., 2018). Ovary is an important reproductive organ in females and goes through a series of biological processes during each estrous cycle. Sow prolificacy is tightly modulated by the complex transcriptional network involving coding and non-coding genes in ovaries (Zhang et al., 2015; Huang et al., 2016; Tang et al., 2018).

Covalently closed circular RNAs (circRNAs) are emerging as a novel class of modulators for gene expression (Li et al., 2018). Now, accumulating work has shed light on the critical roles of circRNAs in gonadal development and reproduction performance in many species (Quan and Li 2018). Next-generation sequencing has revealed that endogenous circRNAs are generally expressed in various kinds of porcine tissues in a spatio-temporally specific manner, including ovaries (Liang et al., 2017). Recent studies revealed that human ovary-derived circRNAs are involved in ovarian aging (Cai et al., 2018), thus we investigated whether circRNAs' profile differs in sows with different litter sizes. 
In this study, a total of six ovaries were selected from multiparous sows with intact prolificacy records, and highthroughput sequencing technology coupled with bioinformatic tools were employed to uncover litter-size-related circRNAs, providing potential candidate loci that may be informative for future pig breeding programs.

\section{MATERIALS AND METHODS}

\section{Ethics Statement and Sample Collection}

This study was approved by the Animal Care and Use Committee in Northwest A\&F University (No. 2018-019). The ovaries in our study were collected from sows 4 days after the fourth delivery picked from a commercial sow piggery in Hanshiwei Food Ltd., Co. (Dahua, Guangxi, China), which is negative for PRRSV (porcine reproductive and respiratory syndrome virus) and PCV (porcine circovirus). For RNA-seq, three ovaries were sampled from each group small and large litter size (8.48 \pm 0.53 piglets/ litter in small litter size and $16.19 \pm 0.43$ piglets/litter in large litter size). In RT-qPCR assay, more ovaries were sampled (8.78 \pm 1.75 piglets/litter $v s .14 .83 \pm 1.61$ piglets/litter, $\mathrm{n}=12$ ). All sows were slaughtered in a standard slaughterhouse in Xinyouxian Livestock Ltd., Co., (Xining, Guangxi, China), and the left ovaries were quickly taken and frozen in liquid nitrogen.

\section{RNA-Seq Assay}

The frozen ovary tissues were homogenized in TRIzol ${ }^{\mathrm{TM}}$ reagent (Invitrogen, Carlsbad, CA, USA), and each sample was quantified using ND-1000 Nanodrop (Thermo Fisher, Wilmington, DE, USA). RNA integrity number (RIN) was analyzed with Agilent 2200 (Agilent, Palo Alto, CA, USA), and RNAs with RIN >7.0 were used for RNA-seq analysis.

The total RNA samples $(3 \mu \mathrm{g})$ were treated with Epicenter Ribo-Zero rRNA removal kit (Illumina, San Diego, CA, USA) to remove ribosomal RNA (rRNA) before cDNA library construction, and then ribosome depleted RNAs were fragmented into 150-200 nt by incubation with divalent cations at $94^{\circ} \mathrm{C}$ for $8 \mathrm{~min}$. The cleaved RNA fragments were reverse-transcribed into first- and second-strand cDNA according to the description of
TruSeq RNA LT/HT sample preparation kit (Illumina, USA). Briefly, the cDNA was treated with End-It DNA End Repair Kit to repair the ends, then modified with Klenow to add an A at the $3^{\prime}$ end, and finally ligated to indexed adapters. The ligated cDNA products were purified and treated with uracil DNA glycosylase to remove the second-strand cDNA. Purified first-strand cDNA was enriched by 13-16 cycles of PCR amplification. The final cDNA libraries were evaluated by Bioanalyzer 2200 (Agilent, Santa Clara, CA) and subjected to sequencing by HiSeq 2000 (Illumina, USA).

miRNA libraries were constructed by Ion Total RNA-Seq Kit v2.0 (Life Technologies), and the sizes were selected by PAGE gel and processed for miRNA sequencing.

\section{Bioinformatic Analysis}

Raw sequencing data were tested by performing FAST-QC (http://www.bioinformatics.babraham.ac.uk/projects/fastqc/) and evaluation metrics including quality scores, distribution of nucleotides, GC content, k-mer frequency, and others. Lowquality bases and $\mathrm{N}$ bases were trimmed from the reads by NGSQCToolkit (v2.3.3), and high-quality clean reads were obtained for subsequent analysis. Clean reads were mapped to the reference genome (Sscrofa 11.1 assembly, http://genome. ucsc.edu) using Hisat2 software.

CIRI was used to identify circRNAs. The alignment results (SAM format) were scanned to search paired chiastic clipping and paired-end mapping signals, as well as GT-AG splicing signals. All the sequences with junction sites were realigned to reference genome using dynamic programming algorithm to ensure the reliability of the putative circRNAs. The total number of reads spanning back-spliced junctions was used as an absolute measure of circRNA abundance.

DEseq2 was used to explore the differentially expressed mRNA among different groups, and the criteria were set as $\mid \log 2$ (fold change) $\mid>1$, FDR $<0.05$. EdgeRSeq was used to explore the differentially expressed circRNA and miRNA between groups, with cutoff of $\mid \log 2$ (fold change) $\mid>1, \mathrm{FDR}<0.05$. Gene ontology (GO) function (http://www.geneontology.org/) and Kyoto Encyclopedia of Genes and Genomes (KEGG, http://www.genome.jp/kegg) pathway of genes of target were annotated.

TABLE 1 | Sequences of primers used in this study.

\begin{tabular}{|c|c|c|}
\hline $\begin{array}{l}\text { Primer li } \\
\text { st }\end{array}$ & Sequence $\left(5^{\prime} \rightarrow 3^{\prime}\right)$ & Product leng \\
\hline circ-ERBIN & F: TTCGACATCCCCAGACATCC R: CAGACATCCGAGACGGAGAA & 218 \\
\hline circ-SNTB2 & F: CAACAATGGAGACCCGTCCT R: TCCTTGGTGCTGTTCTGGTG & 267 \\
\hline circ-TCP1 & F: GGGACCTTAAACGCATTGCTA R: GGTCCAGTIITCAGGGTCTGT & 147 \\
\hline circ-KMT2A & F: AGGAGAACGCAGGCACTाTG R: GGAGGAGGTTCACTGTTGCT & 261 \\
\hline circ-LOC397451 & F: GCTTGCATTGAAAACGGGTCTC R: TCCATTACAGGCAGGACAGTG & 261 \\
\hline circ-NUP98 & F: AGCACAGGGACCAGTCTITC R: AGGCTTCCAGTATTGTTGCTG & 248 \\
\hline circ-SENP2 & F: GGGGAAGAGCAAAGTCATGGA R: TCCGTGTGCCATTACAAGCA & 294 \\
\hline circ-CCDC85A & F: CTGCTAGACTTGACCAGCGTT R: CAAATGTGGGCCAATGGTGAT & 180 \\
\hline circ-CCAR1 & F: CCAACATCAGCAGCCCTTGT R: TGCTGCAATCCGAGTATCCC & 218 \\
\hline ACTB & F: GGACTTCGAGCAGGAGATGG R: AGGAAGGAGGGCTGGAAGAG & 134 \\
\hline
\end{tabular}




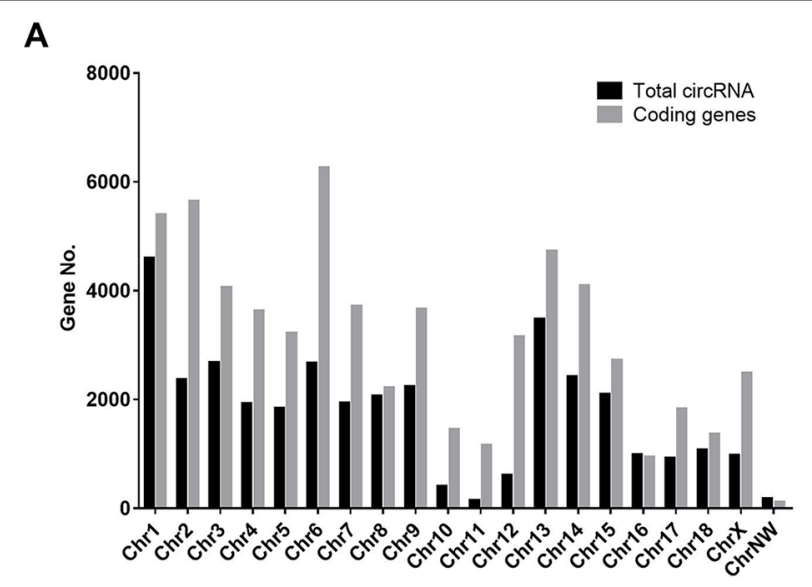

B

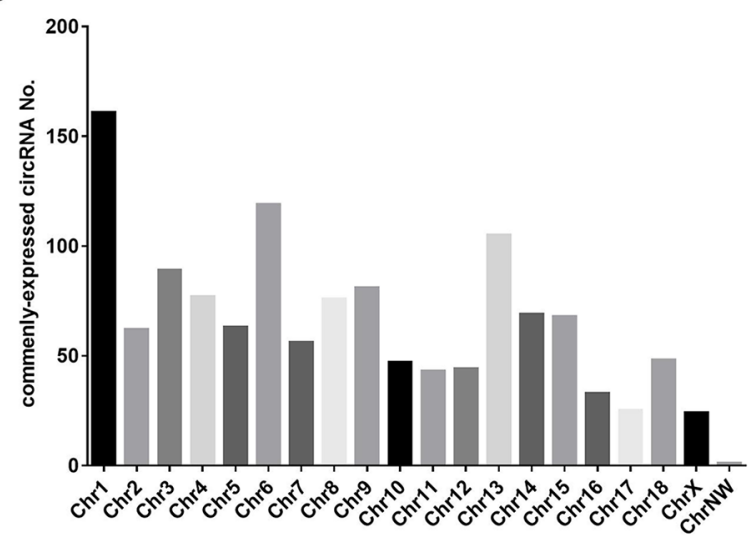

C

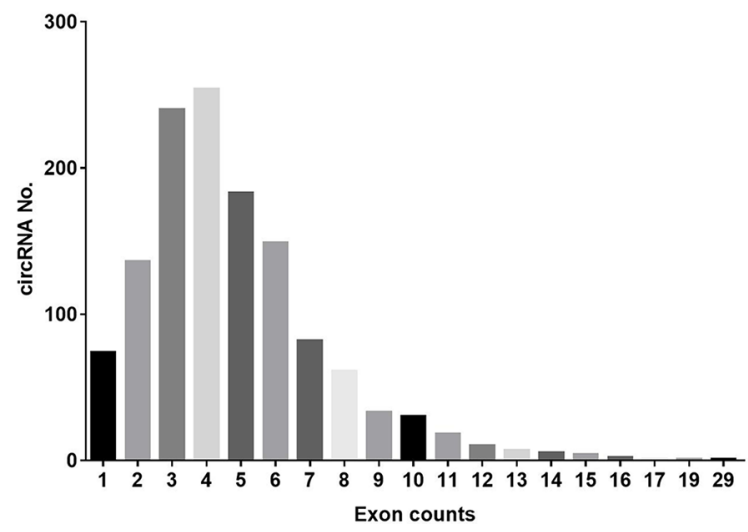

FIGURE 1 | Distribution of predicted circRNAs in our study. (A) The distribution of annotated mRNAs on the genome. (B) The distribution of commenlyexpressed circRNAs on the genome. (C) The exon numbers of commenlyexpressed circRNAs in our study.

\section{CeRNA Network Construction}

The potential miRNA-circRNA interactions were predicted by miRanda (http://miranda.org.uk/) and RNAhybrid2 (http://bibiserv. techfak.uni-bielefeld.de/rnahybrid). The correlation between the expression levels of circRNA and miRNA was calculated with SPSS Pearson correlation assay.

\section{RT-qPCR Verification}

Total RNA was purified using TRIzol ${ }^{\mathrm{TM}}$ reagent (Invitrogen). An aliquot of $2 \mu \mathrm{g}$ total RNA was taken from each sample and reverse transcripted by random primers (TakaRa, Otsu, Japan). For miRNA analysis, specific reverse transcription primers and procedures were used. Real-time PCR reaction $\left(95^{\circ} \mathrm{C} 30 \mathrm{~s}\right.$, then $95^{\circ} \mathrm{C} 5 \mathrm{~s}, 60^{\circ} \mathrm{C} 30 \mathrm{~s}$ for 40 cycles, following $70^{\circ} \mathrm{C} 10 \mathrm{~min}$ for elongation) was performed in triplicate using the Onestep SYBY PrimeScript RT-PCR kit (TakaRa) on a Bio-Rad iQ5 $^{\mathrm{TM}}$ system (Bio-Rad, Berkeley, CA, USA). The expressions of circRNA and miRNA were normalized to that of $A C T B$ and U6 small RNA, respectively. The primer sequences for qPCR are shown in Table 1.

\section{Dual Luciferase Assay}

A $\sim 400$ bp fragment of circRNA containing the putative miRNA binding site was synthesized by General Biosystems (Chuzhou, Anhui, China) and inserted into psiCHECK ${ }^{\mathrm{TM}_{-} 2}$ vector (Promega, Madison, WI, USA) to construct psi-circRNA plasmids. Then the psi-circRNA constructs were co-transfected with their corresponding miRNAs (RiboBio, Guangzhou, China) into 293T cells using Lipofectin ${ }^{\mathrm{TM}} 2000$ (Thermo Fisher Scientific, Waltham, MA, USA), and the luciferase activity was detected by Dual-Luciferase ${ }^{\circledR}$ Reporter Assay System (Promega) $24 \mathrm{~h}$ post transfection.

\section{Statistical Analysis}

Data were processed with SPSS 19.0 software, and results were presented as mean \pm SEM. Significant differences were assessed by unpaired Student's $t$-test and $p<0.05$ was defined as statistical significance.

\section{RESULTS}

\section{Overview of CircRNAs in Porcine Ovary}

Three ovaries in each group (small litter size vs. large litter size) were subjected to RNA sequencing, and a total of 38,722 circRNAs were predicted, which were widely distributed across all chromosomes (Figures 1A, B). However, only 1,291 circRNAs were expressed in all samples (Figure 1C).

\section{Differential CircRNA Expression Profiles in Pigs Differing Litter Size}

In our study, 110 circRNAs [56 down-regulated and 54 up-regulated in larger litter size (LLS)] (Figure 2 A, B) and 20 miRNAs [11 down-regulated and 9 up-regulated in smaller litter size (SLS)] were identified by RNA-seq (Figure 2 C, D).

Given the high variability between samples in RNA-seq, the sample pool was expanded to 12 per group, and a total of 24 ovaries were used when confirming the differentially expressed circRNAs and miRNAs revealed above using RT-PCR. Based on the expanded sample size, RT-PCR assay uncovered that circ-ERBN, 
A

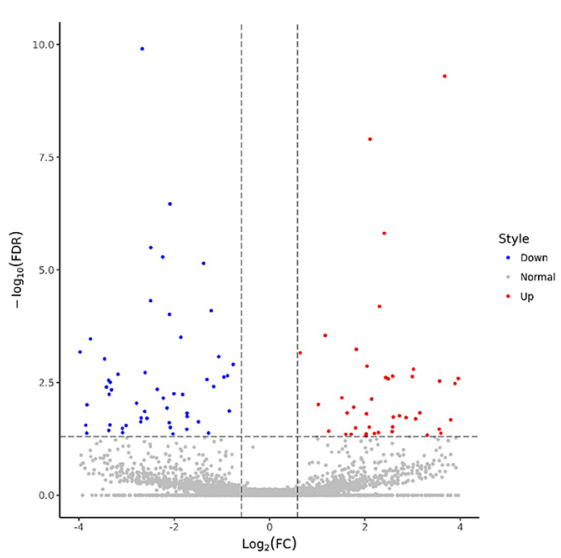

C

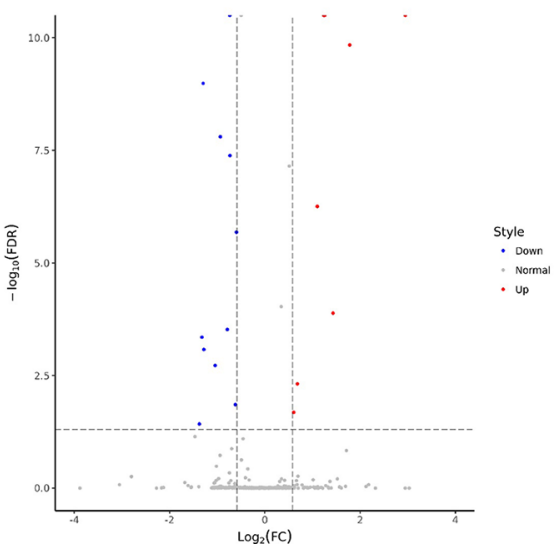

B

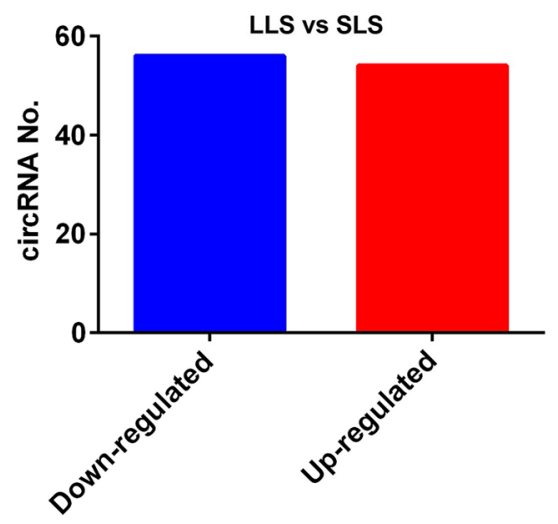

D

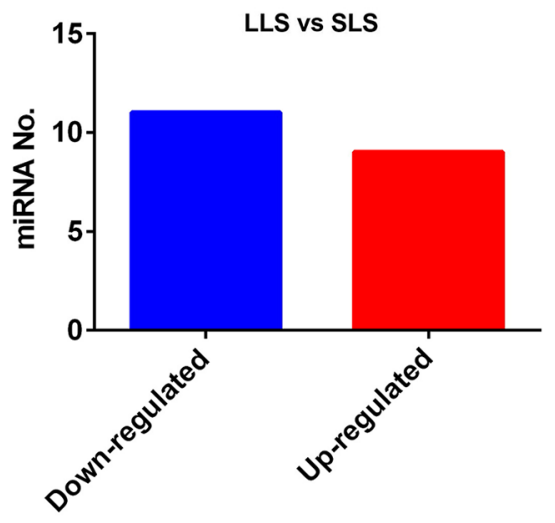

FIGURE 2 | Differentially expressed circRNAs and miRNAs between SLS and LLS pigs. (A) The volcano plot of differentially-expressed circRNAs in LLS and SLS ovaries. (B) The numbers of down- and up-regulated circRNAs in LLS ovaries compared with SLS. (C) The volcano plot of differentially-expressed miRNAs in LLS and SLS ovaries. (D) The numbers of down- and up-regulated miRNAs in LLS ovaries compared with SLS.

circ-SNTB2, circ-TCP1, and circ-KMT2A were significantly higher expressed in porcine ovaries with SLS, while circ-CCDC85A and circ-CCAR1 were significantly higher expressed in porcine ovaries with LLS (Figure 3).

Regarding the differentially expressed miRNAs, the levels of miR-183 and miR-7857-3p were significantly lower in the SLS group while miR-497-5p were significantly lower expressed in pigs with LLS, which were consistent with highthroughput sequencing(Figure 4).

\section{Identification of CircRNA-MiRNA Axis}

Among the differentially expressed circRNAs and miRNAs detected above, RNAhybrid analysis revealed that miR-183 was predicted to interact with circ-TCP1, miR-497 with circCCDC85A. Besides, the expression of miR-183 was reversely correlated with circ-TCP1, and a similar tendency was observed between miR-497 and circ-CCDC85A. Meanwhile, dual-luciferase reporter assay has shown that miR-183 could directly bind to circ-TCP1, while the direct interaction between miR-497 and circ-CCDC85A was not detected in this assay (Figure 5).

\section{Function Analysis of miR-183}

TargetScan and MiRDB were used to predict the potential targets of miR-183, and the common genes presented by these two strategies were subjected to KEGG and GO analysis. KEGG showed that miR-183 might modulate DNA-templated and RNA PolII-mediated transcription (Figure 6A). GO enrichment assay indicated that miR-183 might be tightly related with PI3K-Akt signaling activity (Figure 6B).

\section{DISCUSSION}

The aim of the current study was to identify potential circRNAs related with swine fertility. Even RNA-seq only screened out a total of 1,291 exon-derived circRNAs that expressed in each ovary in our study; there were still several circRNAs that differentially expressed in ovaries with small and large litter size. To overcome the deficiency of great individual variation, the expressions of circRNAs of interest were further validated by RT-qPCR on larger-scale samples. Of note, significantly more circ-TCP1 was detected in porcine ovaries with SLS, and we focus on circ-TCP1 in the subsequent study. 
A

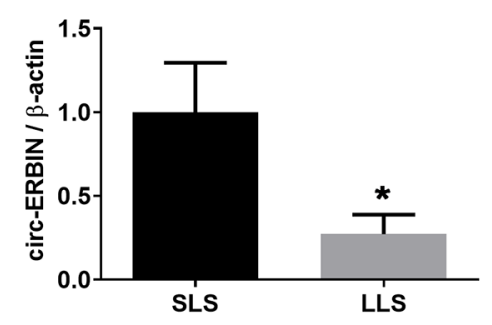

D

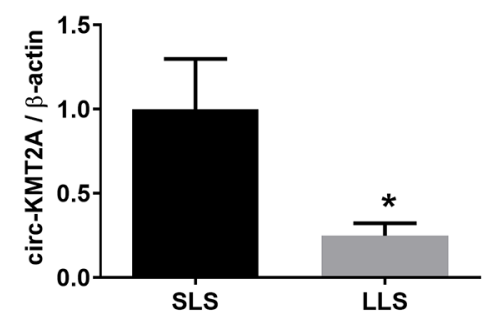

$\mathbf{G}$

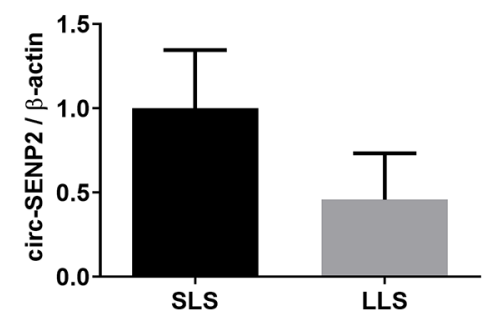

B

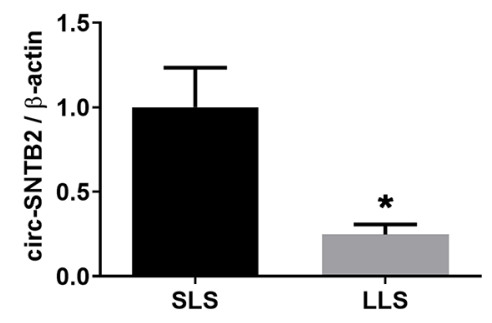

E

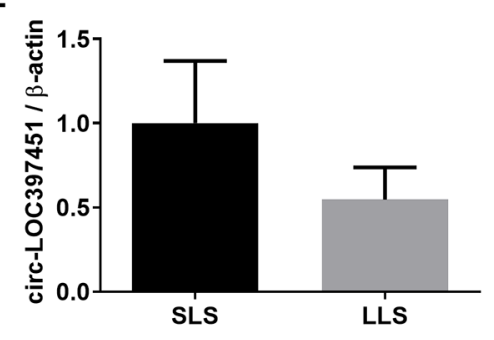

H

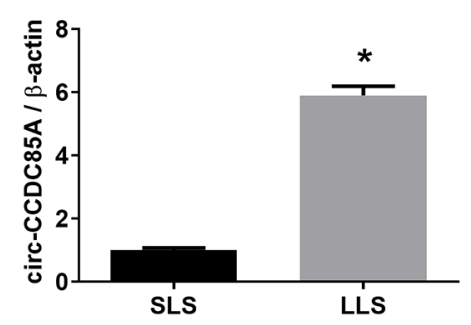

C

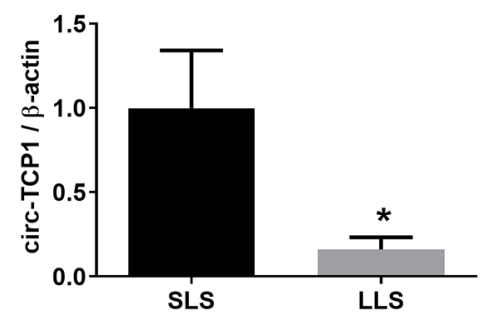

F

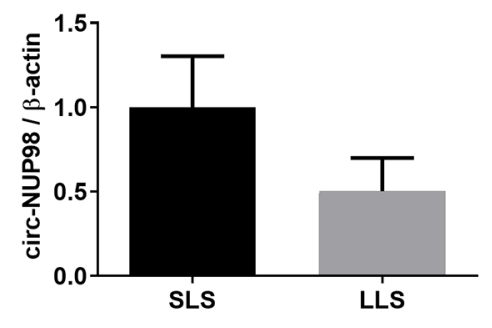

I

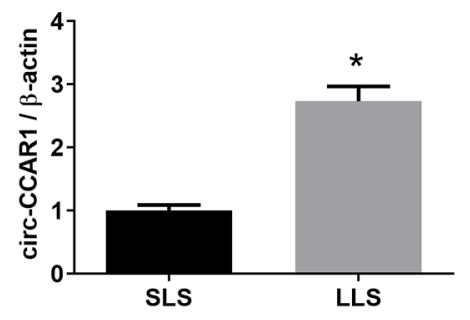

FIGURE 3 | Validation of differentially expressed circRNAs by qPCR. The expression of circ-ERBIN (A), circ-SNTB2 (B), circ-TCP1 (C), circ-KMT2A (D), circLOC397451 (E), circ-NUP98 (F), circ-SENP2 (G), circ-CCDC85A (H) and circ-CCAR1 (I) in SLS and LLS ovaries. " presents $p<0.05$.

One of the well-documented pathways of circRNAs is to competitively bind to functional miRNAs, known as competing endogenous RNAs (ceRNAs) (Li et al., 2018). Here, circ-TCP1, derived from exons 7 and 8 of porcine TCP1 (T-complex protein 1 subunit alpha) gene, was significantly lower expressed in ovaries with LLS. TCP1 gene encodes a molecular chaperone that is a member of the chaperonin containing TCP1 complex (CCT), also known as the TCP1 ring complex (TRiC), which folds various proteins, including actin and tubulin (Sternlicht et al., 1993). Currently, there are no reports about the role of TCP1 in ovary. However, CCT6A, the zeta subunit of CCT, was shown to be expressed in chicken granulosa cells, indicating an important role in folic growth (Wei et al., 2013). Here, circTCP1 was predicted to absorb miR-183 by online RNAhybrid software. Moreover, miR-183 presented a significantly reverse profile with circ-TCP1, and the interaction between circ-TCP1 and miR-183 was further confirmed by luciferase activity assay. Collectively, our data indicated that the circ-TCP1-miR-183 axis might be involved in the biological processes related with litter size.

MiR-183 belongs to the highly conserved miR-183-96182 cluster, which have been shown to be associated with female fertility (Zhang et al., 2019a). Members of miR-18396-182 cluster are known to target the $3^{\prime}$-UTR of FOXO1, an important transcription factor for follicle-stimulating hormone responsive genes in ovarian granulosa cells of rodents (Herndon et al., 2016). FOXO1 and miR-183-96-182 cluster have been also shown to be associated with bovine ovarian follicle development (Zielak-Steciwko and Evans 2016). MiR183 is highly expressed in ovarian cancer cells (Wang et al., 2014; Chen et al., 2016) and down-regulation of miR-183 markedly represses cell proliferation and promotes apoptosis via targeting SMAD family member 4 (Smad4) (Zhou et al., 2019). In our study, KEGG and GO assay suggested that miR183 might be associated with gene transcription, especially related with PI3K-Akt signaling. Genome-wide analysis 
A

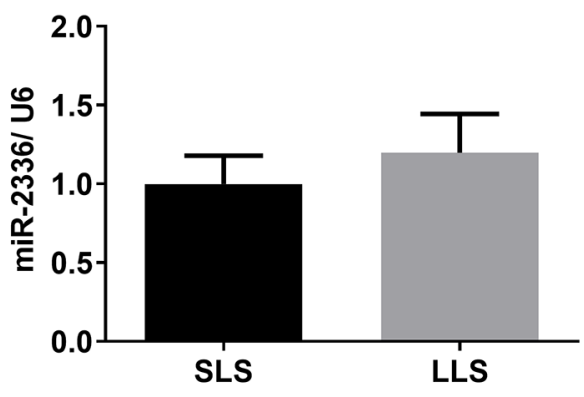

C

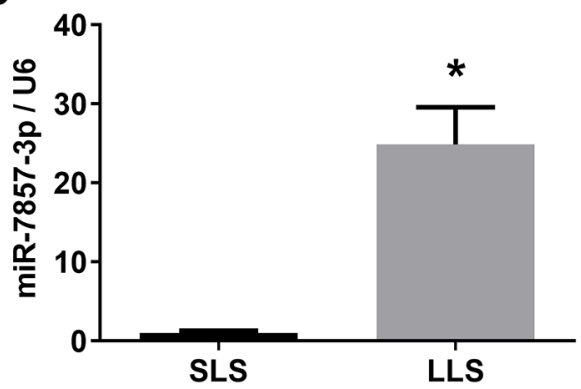

B

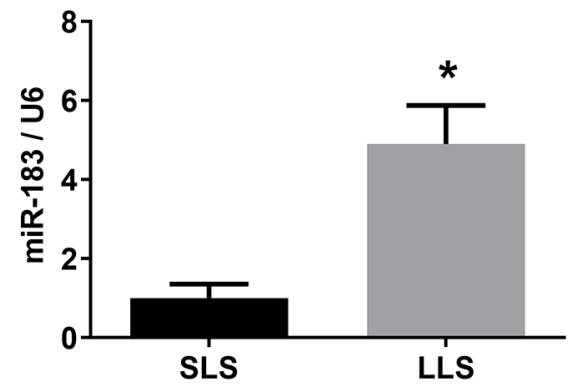

D

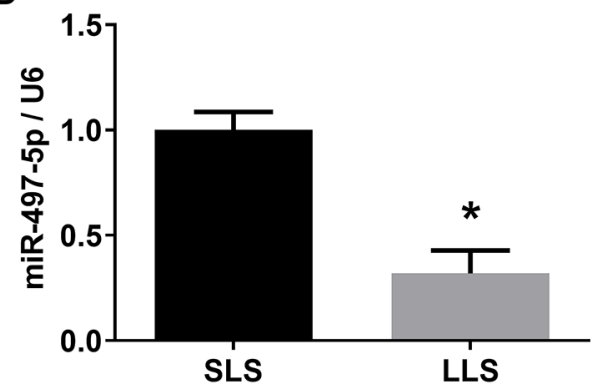

FIGURE 4 | Validation of differentially expressed miRNAs by qPCR. The expression of miR-2336 (A), miR-183 (B), miR-7857 (C) and miR-497-5p (D) in SLS and LLS ovaries. *presents $p<0.05$.

\section{A}

Target: chrs_7598381_7594612_+3769-7CPs

length: 303

IIRHA: $555^{-.0 i R-183}$

length: 23

mfe: $-31.4 \mathrm{kcal} / \mathrm{mol}$

p-value: 1.000000 e+00

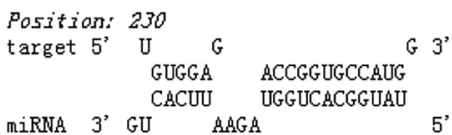

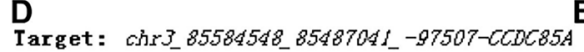

length: 872

HiRHA: $55 c-\bar{a} i R-497$

length: 21

mfe: $-25.0 \mathrm{kcal} / \mathrm{mol}$

$\mathrm{p}$-value: $1.000000 \mathrm{e}+00$

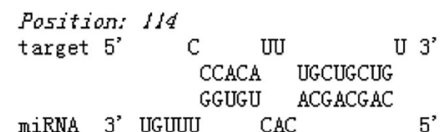

B

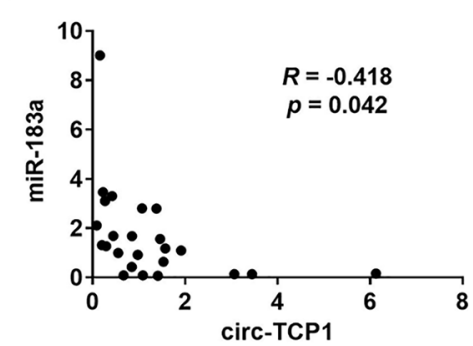

\section{E}

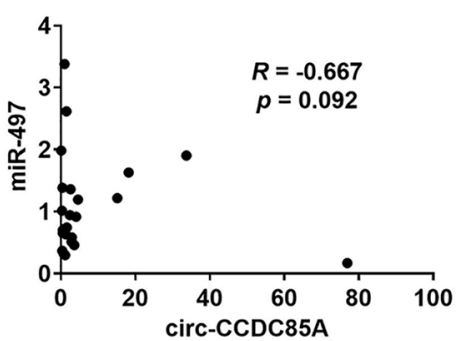

C

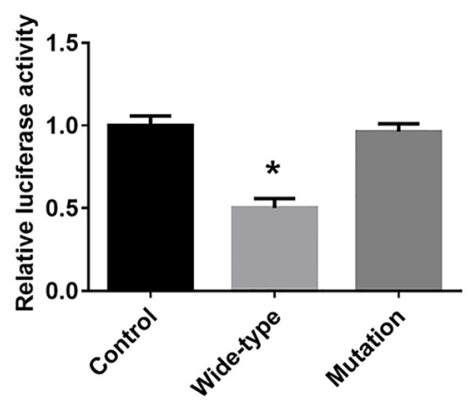

F

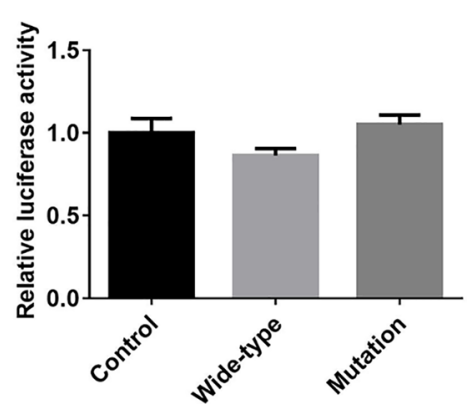

FIGURE 5 | Validation of the interaction between the differentially expressed miRNAs and circRNAs. (A) The putative binding site of miR-183 on circ-TCP1 predicted by RNAhybrid. (B) The correlation of the expression levels between miR-183 and circ-TCP1 in ovaries. (C) The relative luciferase activity of reporter constructs containing wild-type or mutant miR-183-binding sites from circ-TCP1 following cotransfection with control or miR-183 mimics. (D) The putative binding site of miR-497 on circ-CCDC85A predicted by RNAhybrid. (E) The correlation of the expression levels between miR-497 and circ-CCDC85A in ovaries. (F) The relative luciferase activity of reporter constructs containing wild-type or mutant miR-497-binding sites from circ-CCDC85A following cotransfection with control or miR-497 mimics. *presents $p<0.05$. 


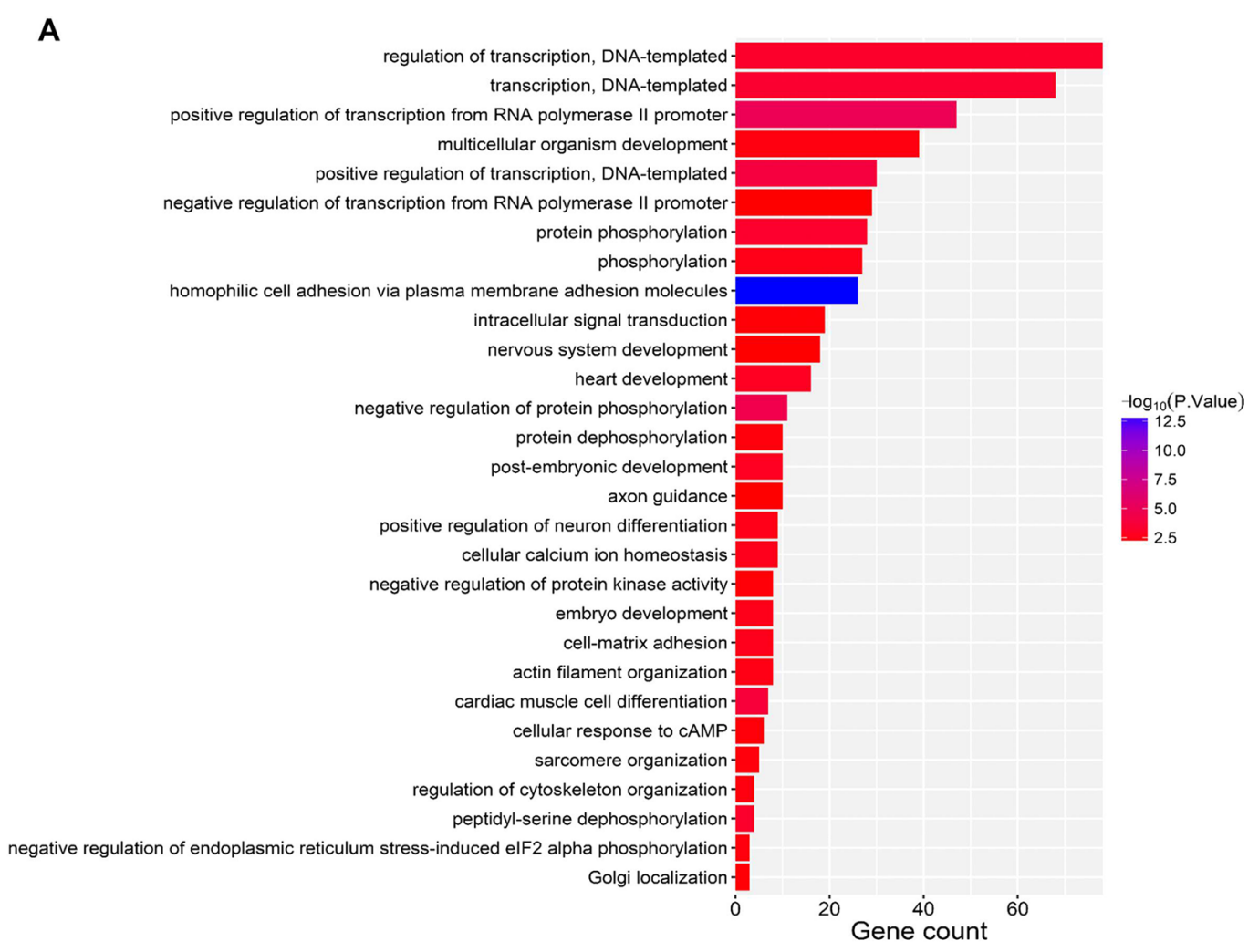

B

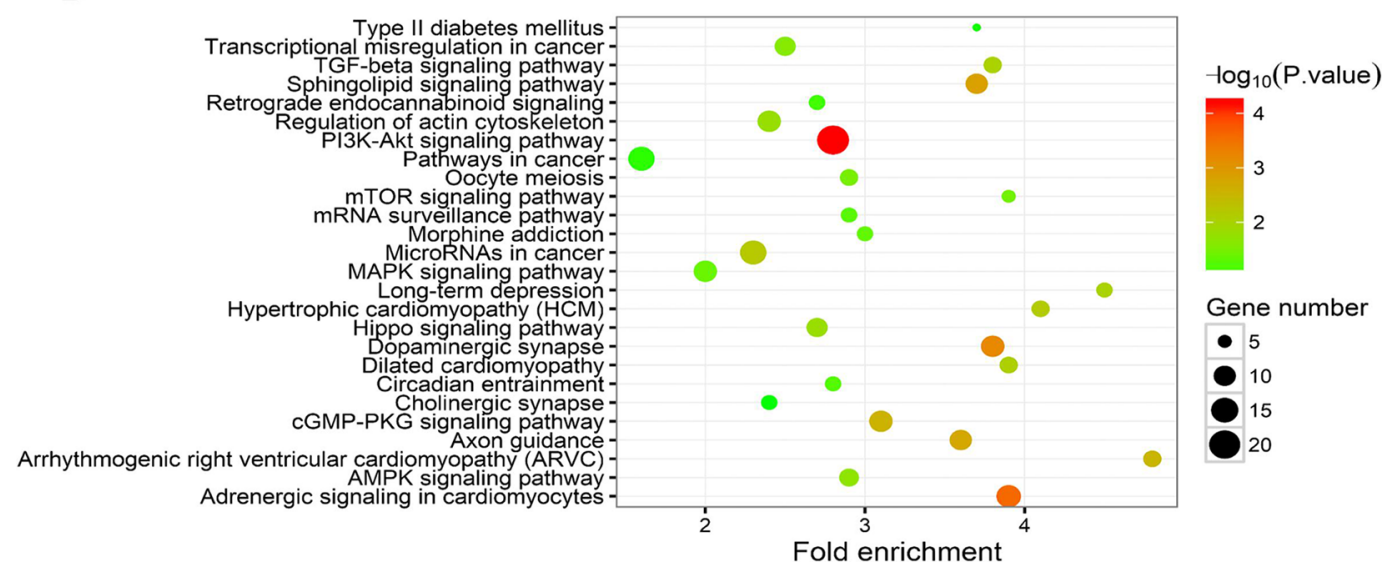

FIGURE 6 | Functional analysis of miR-183. (A) The KEGG pathway analysis of miR-183 target genes. (B) The GO enrichment analysis of miR-183 target genes.

revealed that genes related with PI3K-Akt activity were changed during different follicular stages in the ovaries of Duroc pigs (Liu et al., 2018), and PI3K-Akt activity was significantly inhibited when cell growth of porcine ovarian granulosa was impaired by extracellular stimuli (Wang et al., 2019; Zhang et al., 2019b). Altered PI3K-Akt signaling was also reported to contribute to impeded $17 \beta$-estradiol secretion in ovary cells (Wu et al., 2017). However, the molecular mechanism of
miR-183-PI3K-Akt axis in ovary and their effects on litter size requires further investigation.

\section{CONCLUSION}

In ourstudy, genome-wideidentification of exon-derived circRNAsin porcine ovaries was performed by RNA-seq, and many of which were 
differently expressed in ovaries with variant litter sizes. Furthermore, most exonic circRNAs harbored miRNA binding sites, and circTCP1-miR-183 axis might be associated with swine litter size.

\section{DATA AVAILABILITY STATEMENT}

The data generated in this study has been uploaded to NCBI and can be found under accession number: GSE136592

\section{ETHICS STATEMENT}

This study was approved by Animal Care and Use Committee in Northwest A\&F University.

\section{REFERENCES}

Cai, H., Li, Y., Li, H., Niringiyumukiza, J. D., Zhang, M., et al. (2018). Identification and characterization of human ovary-derived circular RNAs and their potential roles in ovarian aging. Aging 10, 2511-2534. doi: 10.18632/ aging. 101565

Chen, H., Zhang, L., Zhang, L., Du, J., Wang, H., et al. (2016). MicroRNA-183 correlates cancer prognosis, regulates cancer proliferation and bufalin sensitivity in epithelial ovarian caner. Am. J. Transl. Res. 8, 1748-1755.

Herndon, M. K., Law, N. C., Donaubauer, E. M., Kyriss, B., and HunzickerDunn, M. (2016). Forkhead box O member FOXO1 regulates the majority of follicle-stimulating hormone responsive genes in ovarian granulosa cells. Mol. Cell. Endocrinol. 434, 116-126. doi: 10.1016/j.mce.2016.06.020

Huang, L., Yin, Z. J., Feng, Y. F., Zhang, X. D., Wu, T., et al. (2016). Identification and differential expression of microRNAs in the ovaries of pigs (Sus scrofa) with high and low litter sizes. Animal Genet. 47, 543-551. doi: 10.1111/ age. 12452

Kemp, B., Da Silva, C. L. A., and Soede, N. M. (2018). Recent advances in pig reproduction: Focus on impact of genetic selection for female fertility. Reprod. Domestic Animals 53, 28-36. doi: 10.1111/rda.13264

Li, X., Yang, L., and Chen, L.-L. (2018). The biogenesis, functions, and challenges of circular RNAs. Mol. Cell 71, 428-442. doi: 10.1016/j.molcel.2018.06.034

Liang, G., Yang, Y., Niu, G., Tang, Z., and Li, K. (2017). Genome-wide profiling of Sus scrofa circular RNAs across nine organs and three developmental stages. DNA Res. 24, 523-535. doi: 10.1093/dnares/dsx022

Liu, Y., Li, M., Bo, X., Li, T., Ma, L., et al. (2018). Systematic analysis of long non-coding RNAs and mRNAs in the ovaries of Duroc pigs during different follicular stages using RNA sequencing. Int. J. Mol. Sci. 19, E1722. doi: 10.3390/ ijms 19061722

Quan, G., and Li, J. (2018). Circular RNAs: biogenesis, expression and their potential roles in reproduction. J. Ovarian Res. 11, 9-9. doi: 10.1186/ s13048-018-0381-4

Sternlicht, H., Farr, G. W., Sternlicht, M. L., Driscoll, J. K., Willison, K., et al. (1993). The t-complex polypeptide 1 complex is a chaperonin for tubulin and actin in vivo. Proc. Natl Acad. Sci. U. S. A. 90, 9422-9426. doi: 10.1073/pnas.90.20.9422

Tang, L.-T., Ran, X.-Q., Mao, N., Zhang, F.-P., Niu, X., et al. (2018). Analysis of alternative splicing events by RNA sequencing in the ovaries of Xiang pig at estrous and diestrous. Theriogenology 119, 60-68. doi: 10.1016/j. theriogenology.2018.06.022

Wang, L., Zhu, M.-J., Ren, A.-M., Wu, H.-F., Han, W.-M., et al. (2014). A tenmicroRNA signature identified from a genome-wide microRNA expression

\section{AUTHOR CONTRIBUTIONS}

GX conducted the study and drafted the manuscript. $\mathrm{HZ}$ and $\mathrm{XL}$ assisted $\mathrm{t}$ in ovaries sampling and data analysis. JH, GY gave critical comments about experiment design and manuscript drafting. SS supervised the experiment.

\section{FUNDING}

This work was supported by the National Key Technology $\mathrm{R}$ and D Program of China (2015BAD03B01-10).

profiling in human epithelial ovarian cancer. PloS One 9, e96472-e96472. doi: 10.1371/journal.pone.0096472

Wang, W., Luo, S., Ma, J., Shen, W., and Yin, S. (2019). Cytotoxicity and DNA damage caused from Diazinon exposure by inhibiting the PI3K-AKT pathway in porcine ovarian granulosa cells. J. Agric. Food Chem. 67, 19-31. doi: 10.1021/ acs.jafc. 8 b05194

Wei, Q., Zhu, G., Cui, X., Kang, L., Cao, D., et al. (2013). Expression of CCT6A mRNA in chicken granulosa cells is regulated by progesterone. Gen. Comp. Endocrinol. 189, 15-23. doi: 10.1016/j.ygcen.2013.04.019

Wu, C., Yan, D., Lu, D., Han, T., and Zhao, B. (2017). Alteration of the PI3K/Akt signaling pathway by swainsonine affects $17 \beta$-Estradiol secretion in ovary cells. Theriogenology 103, 123-129. doi: 10.1016/j.theriogenology.2017.07.033

Zak, L. J., Gaustad, A. H., Bolarin, A., Broekhuijse, M. L. W. J., Walling, G. A., et al. (2017). Genetic control of complex traits, with a focus on reproduction in pigs. Mol. Reprod. Dev. 84, 1004-1011. doi: 10.1002/mrd.22875

Zhang, J., Xu, Y., Liu, H., and Pan, Z. (2019a). MicroRNAs in ovarian follicular atresia and granulosa cell apoptosis. Reprod. Biol. Endocrinol.: RB\&E 17, 9-9. doi: 10.1186/s12958-018-0450-y

Zhang, T., Sun, X., Li, L., Ma, J., Zhang, R., et al. (2019b). Ochratoxin A exposure impairs porcine granulosa cell growth via the PI3K/AKT signaling pathway. J. Agric. Food Chem. 67, 2679-2690. doi: 10.1021/acs.jafc.8b06361

Zhang, X., Huang, L., Wu, T., Feng, Y., Ding, Y., et al. (2015). Transcriptomic analysis of ovaries from pigs with high and low litter size. PLoS One 10, e0139514. doi: 10.1371/journal.pone.0139514

Zhou, J., Zhang, C., Zhou, B., and Jiang, D. (2019). miR-183 modulated cell proliferation and apoptosis in ovarian cancer through the TGF- $\beta / \mathrm{Smad} 4$ signaling pathway. Int. J. Mol. Med. 43, 1734-1746. doi: 10.3892/ijmm.2019.4082

Zielak-Steciwko, A. E., and Evans, A. C. O. (2016). Genomic portrait of ovarian follicle growth regulation in cattle. Reprod. Biol. 16, 197-202. doi: 10.1016/j. repbio.2016.07.003

Conflict of Interest: The authors declare that the research was conducted in the absence of any commercial or financial relationships that could be construed as a potential conflict of interest.

Copyright (C) $2019 \mathrm{Xu}$, Zhang, Li, Hu, Yang and Sun. This is an open-access article distributed under the terms of the Creative Commons Attribution License (CC $B Y)$. The use, distribution or reproduction in other forums is permitted, provided the original author(s) and the copyright owner(s) are credited and that the original publication in this journal is cited, in accordance with accepted academic practice. No use, distribution or reproduction is permitted which does not comply with these terms. 